\title{
Monitoring Wound Health through Bandages with Passive LC Resonant Sensors
}

Sadaf Charkhabi ${ }^{1}$, Kyle J. Jackson ${ }^{1}$, Andee M. Beierle ${ }^{1}$, Adam R. Carr ${ }^{1}$, Eric M. Zellner ${ }^{2}$, Nigel F. Reuel $^{1 *}$

${ }^{1}$ Department of Chemical and Biological Engineering, lowa State University, Ames, IA, 50011

${ }^{2}$ Department of Veterinary Clinical Sciences, lowa State University, Ames, IA, 50011

*Corresponding Author - reuel@iastate.edu

\section{Contents}



2. MATLAB Code for Detecting the Resonance Feature ....................................................................

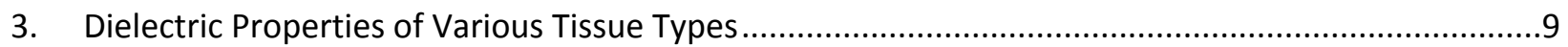

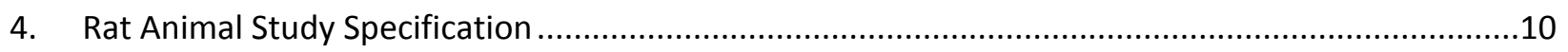

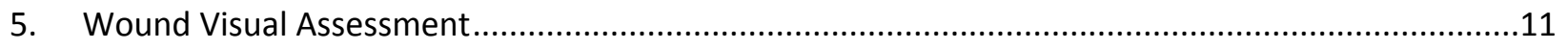

6. Sensor Response Comparison between the Rats .......................................................................

7. Effect of Treatment Method on Wound Healing Rate..................................................................15



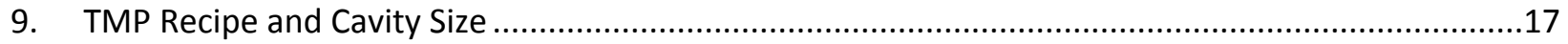

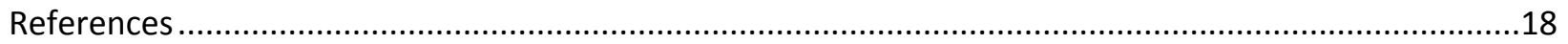




\section{MATLAB Code for Recording Scattering Parameters}

A two-loop reader antenna, each with $54 \mathrm{~mm}$ diameter, were embedded in a 3D-printed plastic cast with $26.7 \mathrm{~mm}$ overlap. The coils were connected to a Copper Mountain Technologies TR1300 two-port vector network analyzer (VNA) with RF-shielded BNC cables. The center of the resonator was aligned to the center of the reader antenna for all tests performed in this paper. The phase and the magnitude data of transmission (S21) and reflection scattering parameters $\left(S_{11}\right)$ in frequency range of $0-250 \mathrm{MHz}$ were automatically recorded by connecting the VNA to a Lenovo laptop using the following MATLAB script:

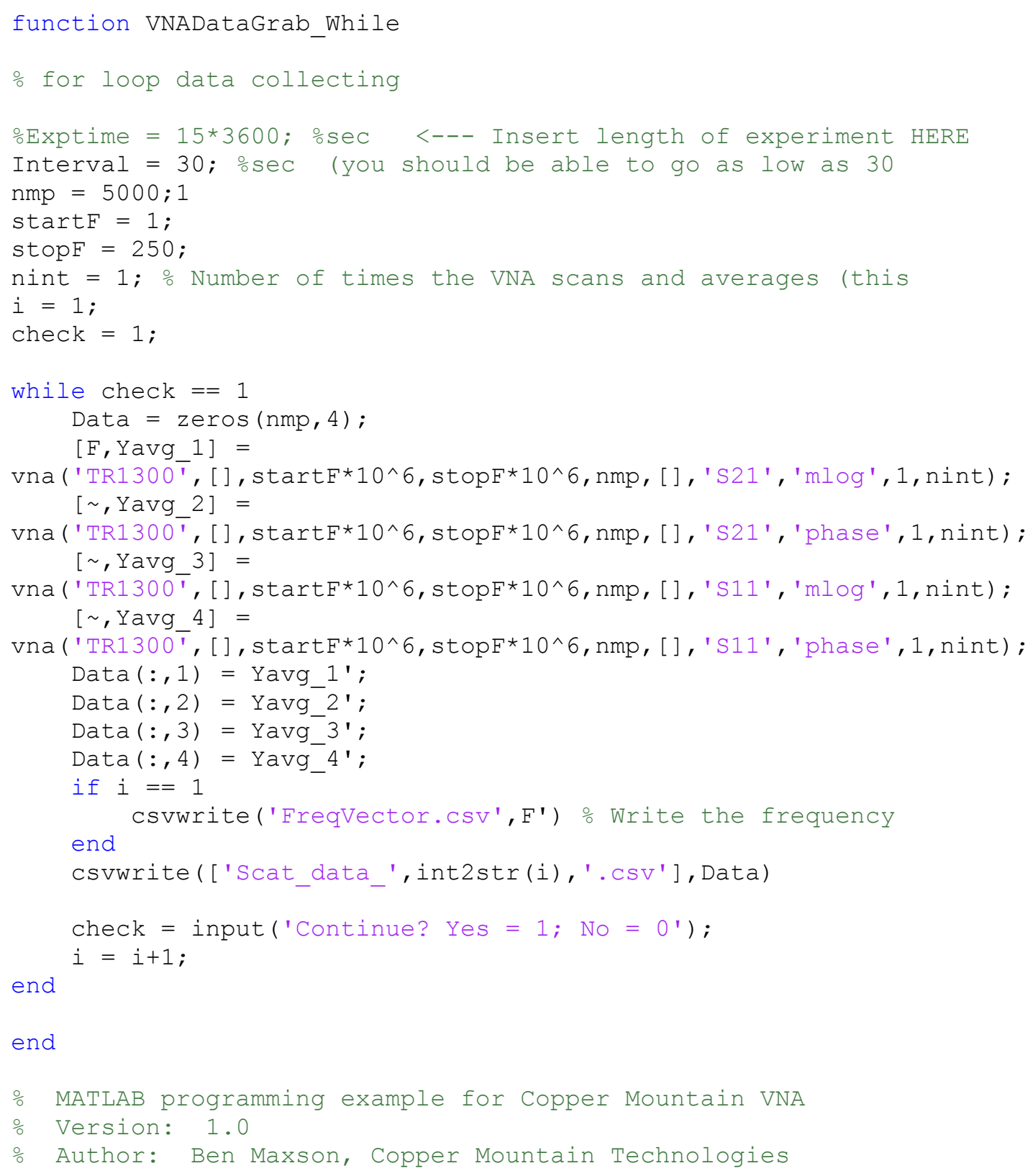




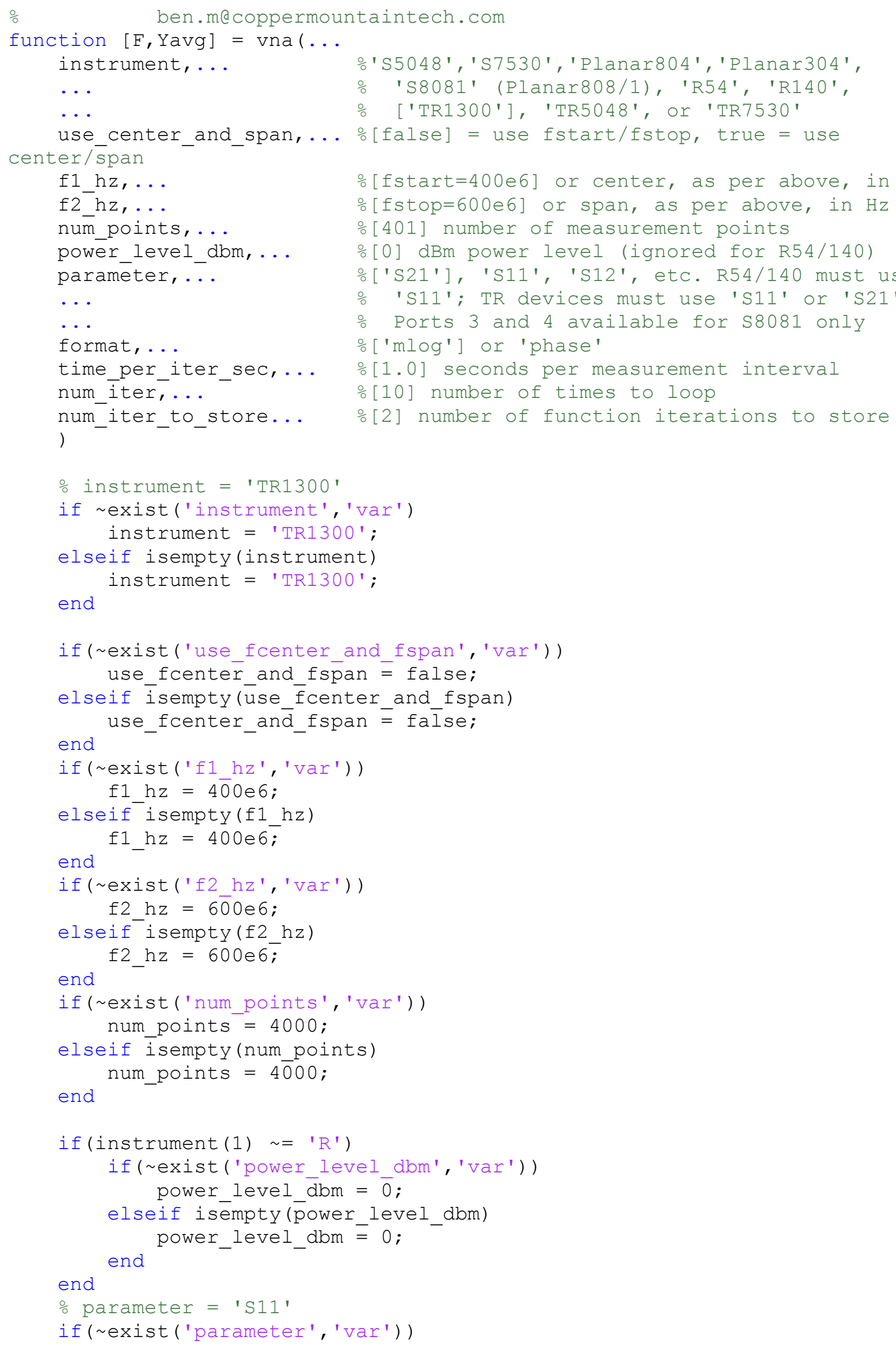









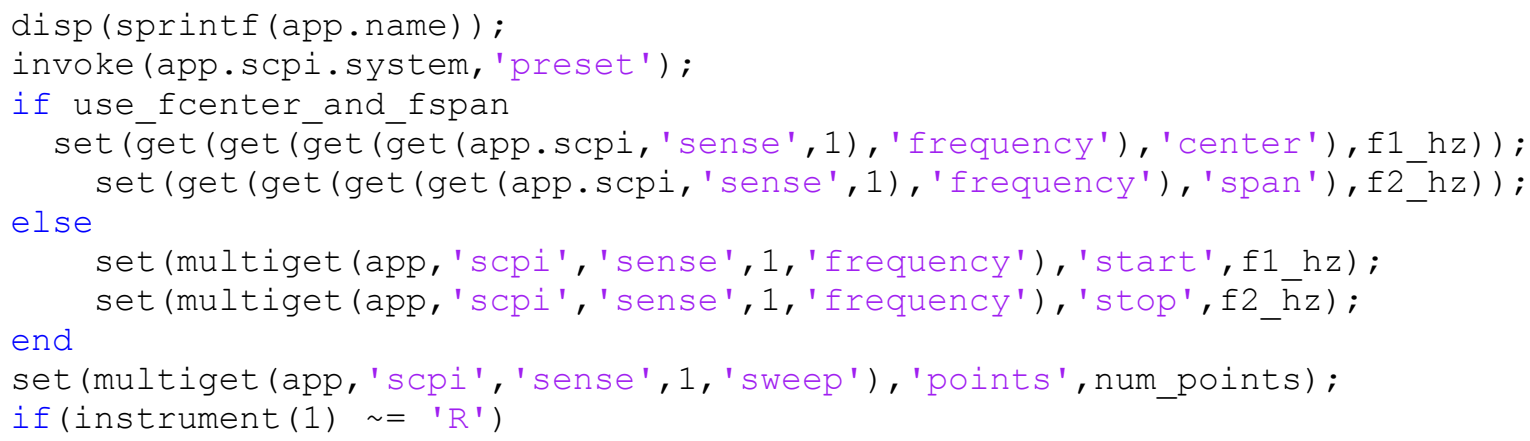




\section{MATLAB Code for Detecting the Resonance Feature}

The magnitude of forward transmission scattering parameter response $\left(S_{21}(d B)\right)$ recorded via python script mentioned above is further analyzed using MATLAB code to automatically find the resonant and anti-resonant frequencies and their corresponding $S_{21}(d B)$ values. The sensor response is reported as resonant frequency $(\mathrm{MHz})$ and peak-to-peak amplitude $(\mathrm{dB})$ which is the difference between resonant and anti-resonant $S_{21}(d B)$ values.

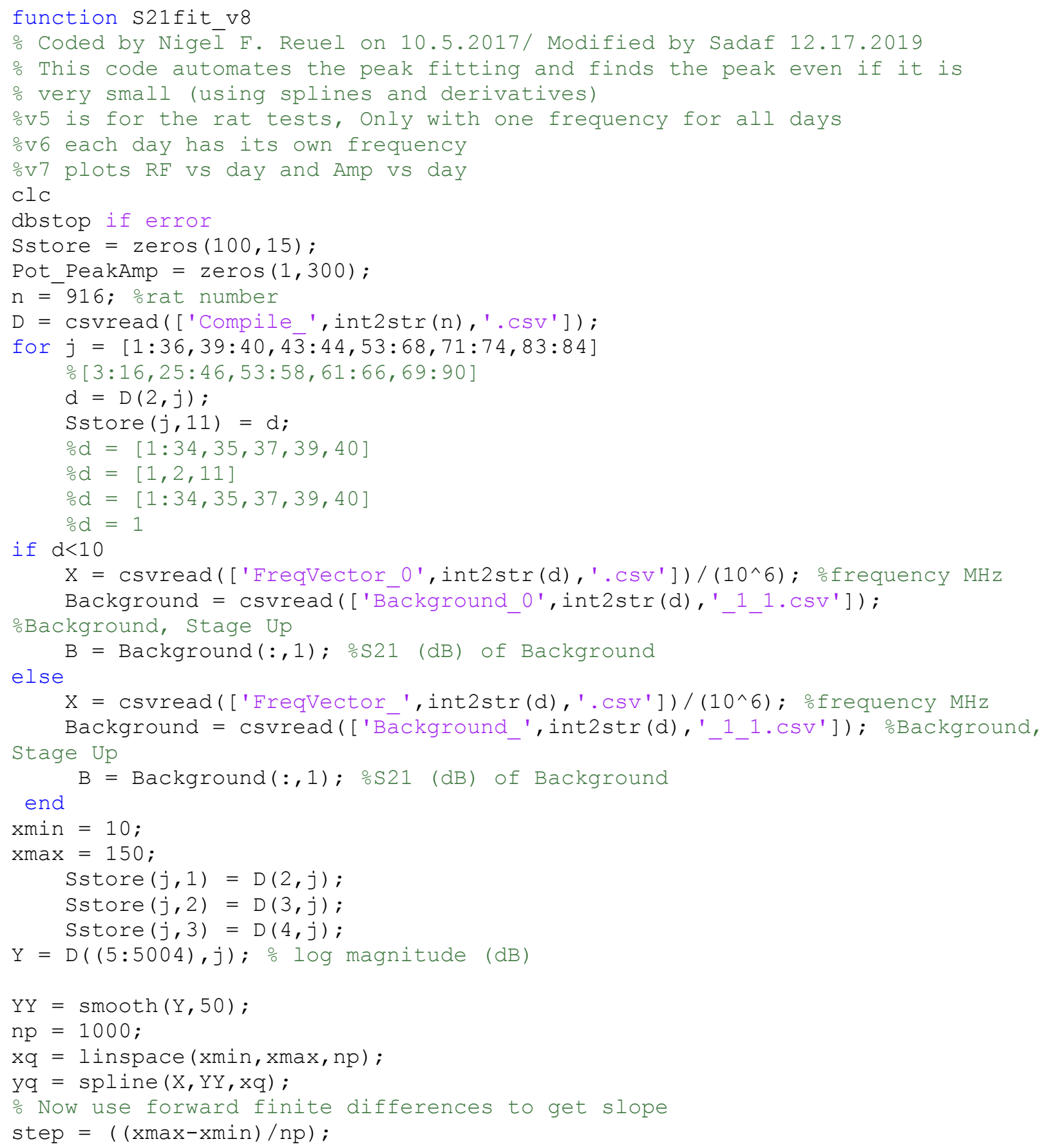














\section{Dielectric Properties of Various Tissue Types}

The sensor response in terms of shift in the resonant frequency and change in the peak-to-peak amplitude is attributed to variations in the relative permittivity and conductivity of the tissues involved in the wound healing. These dielectric properties for muscle, fat, wet skin, and blood are demonstrated in Table S1 for tissue mimicking phantoms (TMPs) at $2.45 \mathrm{GHz}$ and real tissue at $100 \mathrm{MHz}$.

Table S1. Relative permittivity and conductivity of various tissue.

\begin{tabular}{cccccccc}
\hline \multirow{2}{*}{ Tissue } & \multicolumn{2}{c}{ TMP @ $\mathbf{2 . 4 5} \mathbf{~ G H z}^{\mathbf{1}}$} & \multicolumn{2}{c}{${\text { IT'IS Database @ } \mathbf{1 0 0 ~} \mathbf{~ M H z}^{\mathbf{2}}}^{2}$} & \multicolumn{2}{c}{ Real Tissue @100MHz } \\
\cline { 2 - 8 } & Permittivity & Conductivity(S/m) & Permittivity & Conductivity(S/m) & Permittivity & Conductivity(S/m) \\
\hline Muscle & 52.8 & 1.71 & 66 & 0.78 & 60 & 0.7 \\
\hline Fat & 5.24 & 0.10 & 6.07 & 0.036 & 5.5 & 0.02 \\
\hline Skin & 42.92 & 1.56 & 72.9 & 0.49 & 60 & 0.5 \\
\hline Blood & 58.35 & 2.50 & 76.8 & 1.23 & 75 & 1.2 \\
\hline
\end{tabular}




\section{Rat Animal Study Specification}

The animal study experiment was performed on 12 Wistar 112 line rats with a $2 \mathrm{~cm} \times 2 \mathrm{~cm}$ square wound which was created surgically by removing the skin and the subcutaneous fat layer at the beginning of the study. The rats were then randomly divided into two treatment groups (Hydrogel vs. VEGF) and then randomly divided again for Telfa/No Telfa studies.

Table S2. Rat study specifications.

\begin{tabular}{|c|c|c|c|c|}
\hline Rat \# & Treatment & Layer & RF - Significant difference & Amp - Significant difference \\
\hline 905 & Hydrogel & Telfa & 1 & 1 \\
\hline 906 & Hydrogel & No Telfa & 0 & 0 \\
\hline 907 & VEGF & Telfa & 1 & 1 \\
\hline 908 & VEGF & Telfa & 1 & 0 \\
\hline 909 & Hydrogel & No Telfa & 0 & 1 \\
\hline 910 & VEGF & No Telfa & 0 & 0 \\
\hline 911 & VEGF & No Telfa & 0 & 0 \\
\hline 912 & VEGF & No Telfa & 0 & 0 \\
\hline 913 & Hydrogel & Telfa & 1 & 1 \\
\hline 914 & Hydrogel & Telfa & 1 & 1 \\
\hline 915 & VEGF & Telfa & 1 & 0 \\
\hline 916 & Hydrogel & No Telfa & 1 & 1 \\
\hline
\end{tabular}




\section{Wound Visual Assessment}

In addition to measuring the resonant frequency and the peak-to-peak amplitude of the resonant sensor, the wounds were visually assessed during each bandage change. By taking a photograph. The size and the RGB color model values were extracted using the following MATLAB code.

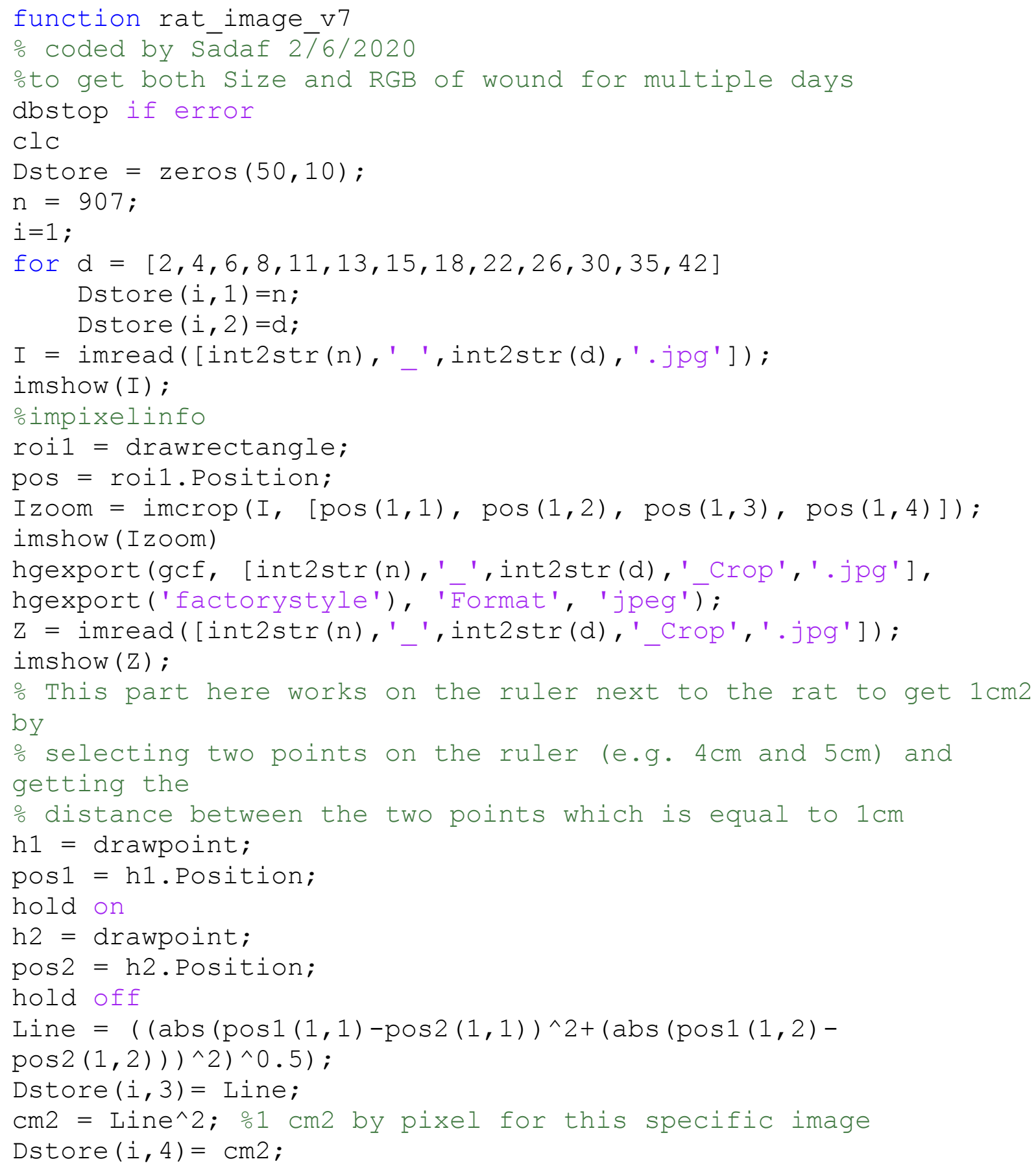




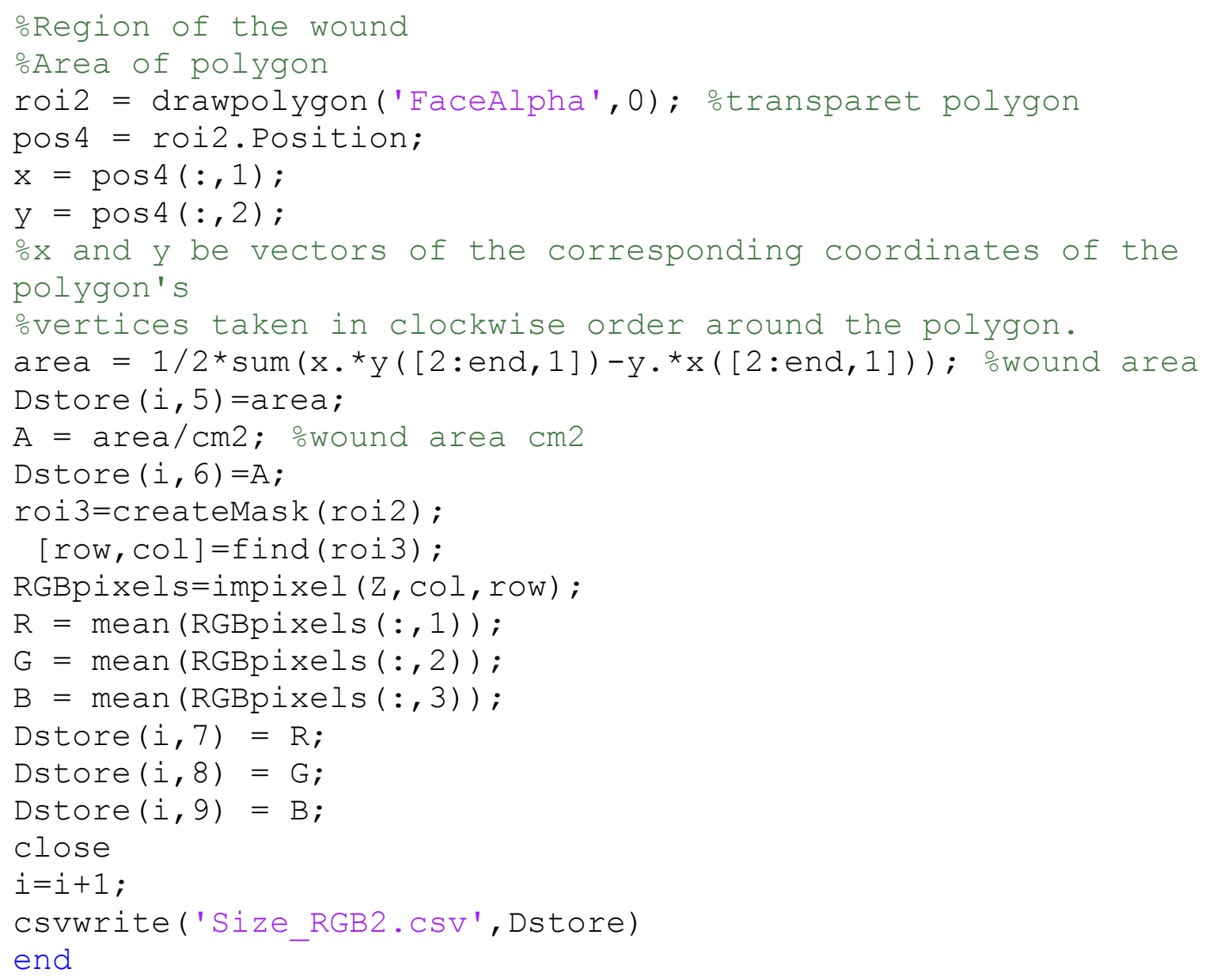




\section{Sensor Response Comparison between the Rats}

In order to study the applicability of resonant sensors for wound monitoring, a small cohort of 12 Wistar rats with an administrated wound of approximately $2 \mathrm{~cm} \times 2 \mathrm{~cm}$ on their dorsal thorax were used. The rats were divided into four different groups to investigate the effect of treatment method (VEGF vs. hydrogel) as well as the wound dressing layers on the healing rate and the sensor response. Due to the variations in the initial size of the administrated wound, the unique healing rate, and the wound dressing layer between the rats, analyzing the longitudinal results of the pooled animal data is not readily clear (Figure S1). Therefore, each rat was individually analyzed, comparing the wound response to the internal control (healthy tissue).

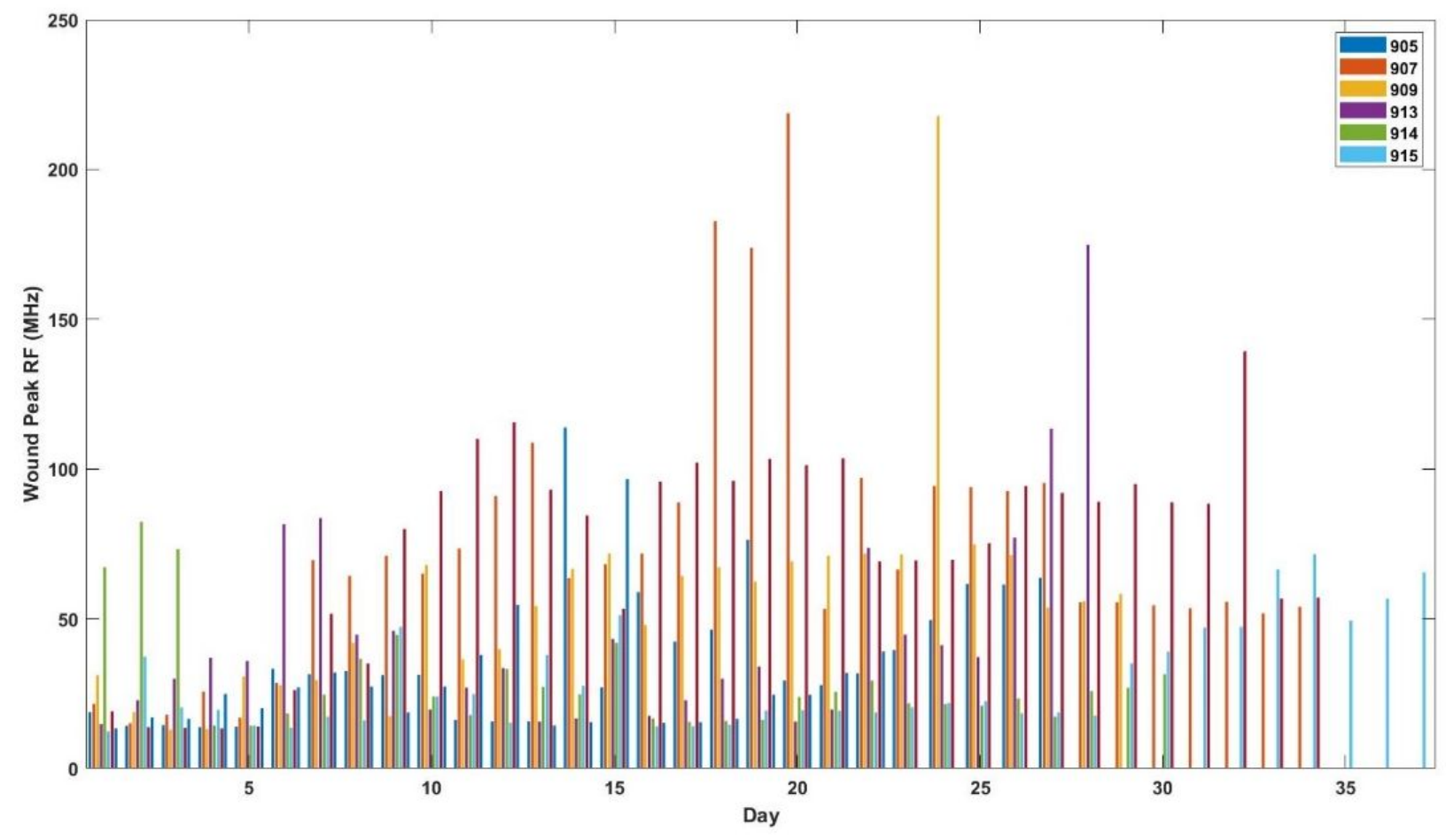




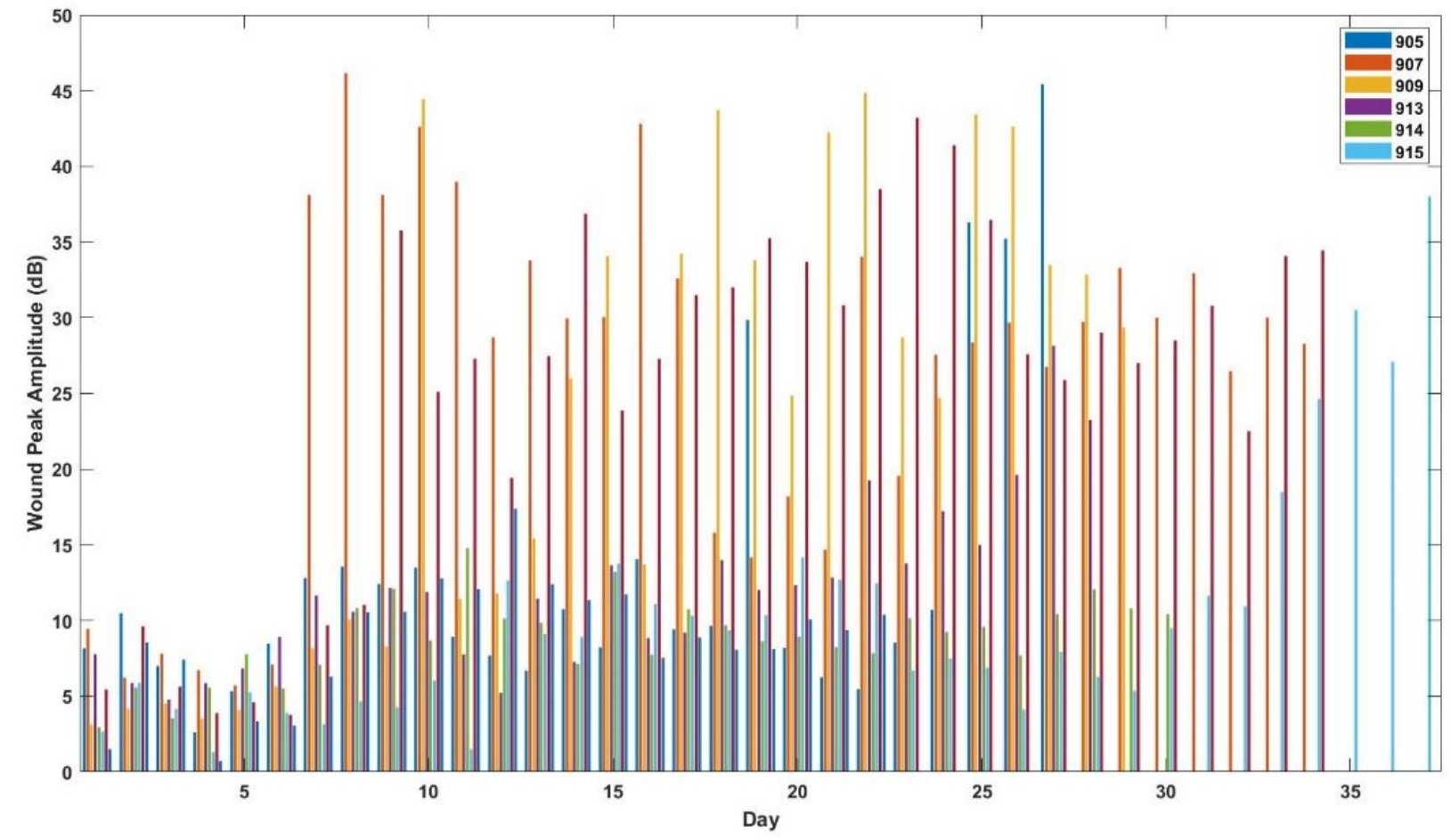

Figure S1. Bar plots comparing the peak resonant frequency and the peak-to-peak amplitude for the sensor placed on the wound area of the rats 905, 907, 909, 913, 914, 915. 


\section{Effect of Treatment Method on Wound Healing Rate}

In order to investigate the effect of treatment method on the rate of wound closure, the rats were randomly divided into two group to be treated with either Hydrogel or VEGF. There was not a noticeable dependency on the treatment method observed for the resonant frequency and the peak-to-peak amplitude; however, VEGF slightly improved the wound healing rate.

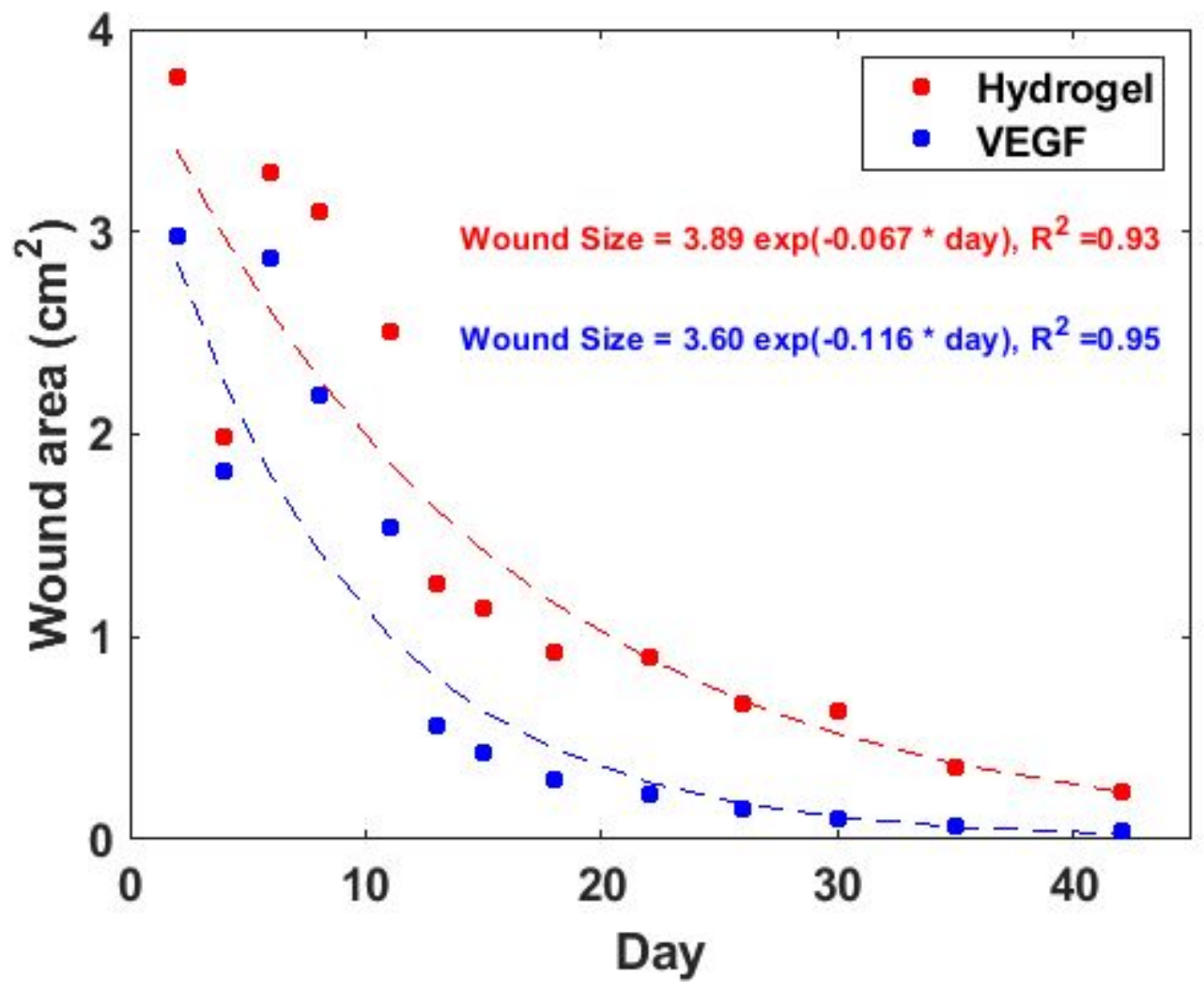

Figure S2. Effect of treatment method on the wound closure.

The wound area was fit to the time using decaying exponential fit for both hydrogel $\left(R^{2}=0.93\right)$ and VEGF $\left(R^{2}=0.95\right)$. The rate of healing, defined as the first derivative of wound size vs. time function is demonstrated for Hydrogel (Eq. S1) and VEGF (Eq. S2). VEGF can slightly improve the healing rate.

$$
\begin{aligned}
& \frac{d y}{d x}=-0.26 \exp (-0.067 x) \\
& \frac{d y}{d x}=-0.42 \exp (-0.116 x)
\end{aligned}
$$




\section{Sensor Parameters}

In order to extract the sensor parameters, the sensor response in terms of resonant frequency is plotted as a function of wound closure in rat 915 (Figure S3). The sensor has a linear response after $30 \%$ of wound closure, which is again due to larger experimental noise at the early stages of closure. In the range of $30-65 \%$ wound closure, the sensor response is fit well with a linear function with $\mathrm{R}^{2}=0.94$. In this case, the sensitivity of the sensor is $3.547 \mathrm{MHz} /$ Wound closure\%. Currently, the sensor does not have a repeatable response, which is due to the experimental errors such as inconsistencies in the process of scanning the sensors and reader-sensor misalignments. As discussed in the manuscript, these issues can be addressed in future studies by using a hand-held portable VNA which can make the process of scanning the sensors more consistent. Moreover, including positional cues to correct alignment could also improve quality. It is worth to mention that at this step of sensor animal study, the above-mentioned sensor parameters are specific to rat 915, which was treated with VEGF and had the Telfa separating layer, and cannot be expanded to other rats. With further improving the sensor fabrication method, wound creation, and scanning techniques, the sources of errors which were determined in this initial study will be limited which will ultimately help with generating a global calibration curve.

a)

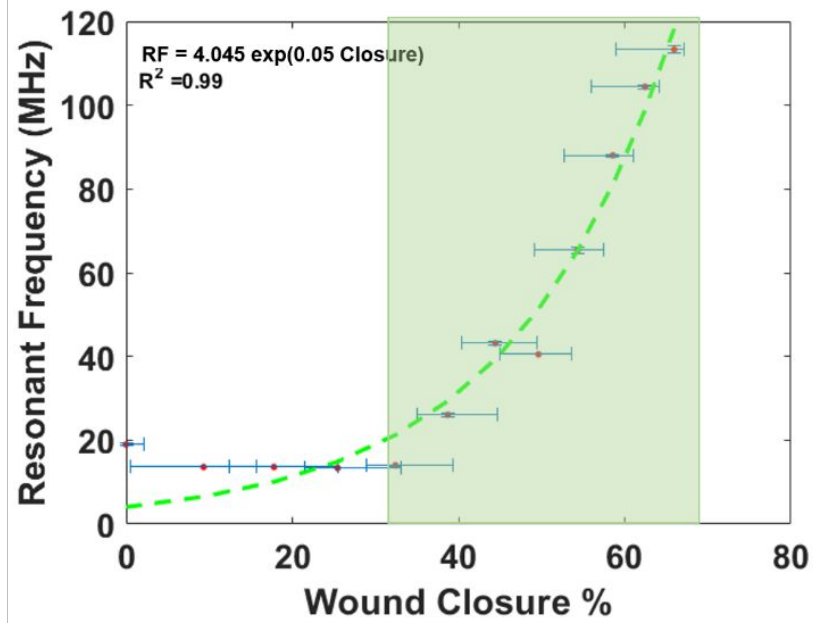

b)

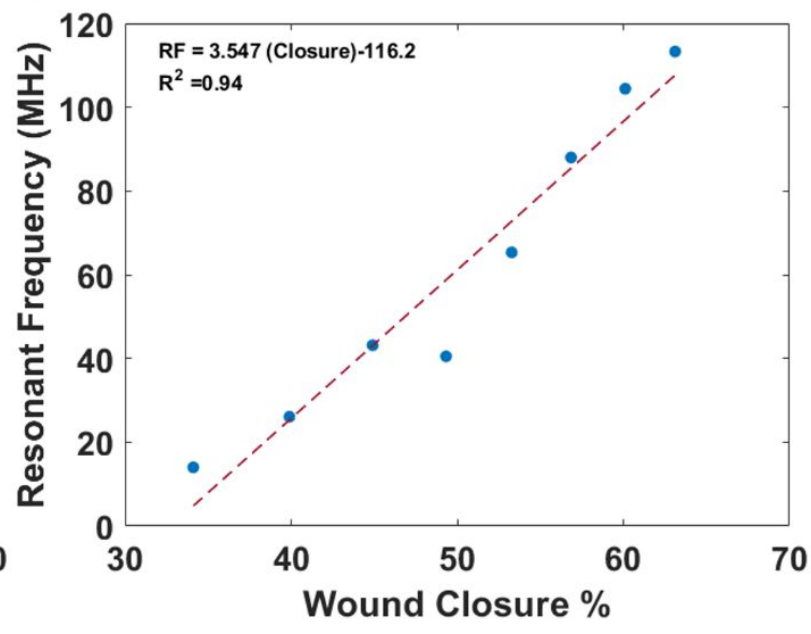


Figure S3. a) Sensor resonant frequency response to wound closure in rat 915; b) linear region of the sensor response.

\section{TMP Recipe and Cavity Size}

Gelatin-based TMPs were prepared using recipe provided by Yilmaz et. al. ${ }^{1}$

Table S3. Ingredients for tissue mimicking phantoms

\begin{tabular}{ccccc}
\hline Ingredient (g) & Wet Skin & Fat & Blood & Muscle \\
\hline Water & 230 & 57.4 & 230 & 230 \\
\hline Gelatin & 34.1 & 15 & 34.1 & 34.1 \\
\hline $\mathrm{NaCl}$ & 1.4 & 0 & 1.2 & 1.2 \\
\hline Oil & 75 & 329.6 & 15 & 35 \\
\hline Detergent & 40 & 10 & 40 & 40 \\
\hline
\end{tabular}

The size of the cavities made in the TMPs to replicate various stages of wound healing is as follow:

Table S4. Cavity size representing wound in TMPs.

\begin{tabular}{ccccc}
\hline Sample & $\mathbf{X}(\mathbf{m m})$ & $\mathbf{Y}(\mathbf{m m})$ & $\mathbf{Z}(\mathbf{m m})$ & Blood Volume $(\boldsymbol{\mu l})$ \\
\hline $\mathbf{1}$ & 3 & 3 & 3 & 27 \\
\hline $\mathbf{2}$ & 4.5 & 4.5 & 4.5 & 91 \\
\hline $\mathbf{3}$ & 7.1 & 7.1 & 7.1 & 360 \\
\hline $\mathbf{4}$ & 14.2 & 14.2 & 10 & 1864 \\
\hline $\mathbf{5}$ & 18.7 & 18.7 & 10 & 3500 \\
\hline $\mathbf{6}$ & 25 & 25 & 15 & 9000 \\
\hline
\end{tabular}


References

(1) Yilmaz, T.; Foster, R.; Hao, Y. Broadband Tissue Mimicking Phantoms and a Patch Resonator for Evaluating Noninvasive Monitoring of Blood Glucose Levels. IEEE Trans. Antennas Propag. 2014, 62, 3064-3075.

(2) Hasgall, P. A.; Neufeld, E.; Gosselin, M. C.; Klingenböck, A.; Kuster, N.; Hasgall, P.; Gosselin, M. IT'IS Database for Thermal and Electromagnetic Parameters of Biological Tissues. 2012.

(3) Gabriel, S.; Lau, R. W.; Gabriel, C. The Dielectric Properties of Biological Tissues: Ill. Parametric Models for the Dielectric Spectrum of Tissues. Phys. Med. Biol. 1996, 41, 2271. 